\title{
3. Die Preußische Zentralgenossenschaftskasse
}

Als das preußische Finanzministerium am 12. Januar 1928 dem Amtlichen Preußischen Pressedienst die Mitteilung machte, daß der Geheime Finanzrat Carl Semper, bisheriger Präsident der Preußenkasse, bis auf weiteres beurlaubt und Rechtsanwalt Klepper „mit der kommissarischen Leitung des Direktoriums der Preußischen Zentralgenossenschaftskasse beauftragt" worden sei, wußten nicht nur Eingeweihte, daß sich hinter den dürren Worten eine Menge Zündstoff verbarg. Die amtliche Mitteilung konnte dies nur versteckt andeuten: „Der finanzielle Status der Anstalt gibt, wie bereits festgestellt worden ist, zu irgendwelchen Besorgnissen keinen Anlaß. Das preußische Staatsministerium ist überdies fest entschlossen, alle erforderlichen Maßnahmen zu treffen, um die Preußische Zentralgenossenschaftskasse als leistungsfähiges Kreditinstitut für das landwirtschaftliche Genossenschaftswesen ${ }^{1}$ und den gewerblichen Mittelstand zu erhalten und weiter auszubauen." 2

Es war kein Zweifel, die Preußenkasse war in eine Krise geraten. Die Ursachen dafür waren vielfältig. Zum einen waren sie bereits in der Gründung der Institution angelegt, zum anderen hingen sie mit der besonderen politischen und wirtschaftlichen Situation Preußens nach dem Ersten Weltkrieg und dem sich daraus entwickelnden Gegensatz zum Reich zusammen, und sie waren schließlich, drittens, die Folge davon, daß sich die Leitung von Geheimrat Semper und seinem Stab den neuen wirtschaftlichen und sozialen Bedingungen nach dem Ersten Weltkrieg und vor allem nach der Inflation 1923 nicht mehr gewachsen zeigte. Diese drei Ursachen müssen genauer untersucht werden, will man die harten Auseinandersetzungen auf dem Agrarsektor, die zwischen der Preußenkasse und den Genossenschaften und zwischen Preußen und dem Reich in der Endphase der Republik ausgetragen wurden, verstehen.

„Die Konstruktion dieser Großbank des kleinen Mannes" wurde von zwei sehr unterschiedlichen Überlegungen beeinflußt, einmal von rein banktechnischen, zum anderen von sozialpolitischen Erwägungen ${ }^{3}$. Die banktechnische Notwendigkeit ergab sich aus der Tatsache, daß die Kreditbedürfnisse des Handwerks und der Landwirtschaft sowohl vom zeitlichen Rahmen wie von der Kreditwürdigkeit her besondere waren, die die 1875 gegründete Reichsbank nicht zu befriedigen vermochte, abgesehen davon, daß der Geschäftsbetrieb der Genossenschaften sich kaum mit den normalen Bankgeschäf-

\footnotetext{
${ }^{1}$ Nach der Einteilung von K. Kluthe, S. 21, muß zwischen landwirtschaftlichen Genossenschaften (incl. der ländlichen Spar- und Darlehenskassen), gewerblichen Genossenschaften (incl. Volksbanken), Konsum- und Wohnungsbaugenossenschaften unterschieden werden. Die Genossenschaften haben sich im Laufe des 19. Jahrhunderts, anknüpfend an historische Modelle, gebildet, um auf dem Wege der Selbsthilfe Waren zu produzieren und zu verteilen. Die beiden wichtigsten Genossenschaftsgründungen waren die von Hermann Schulze-Delitzsch (1808-1883) und Friedrich Wilhelm Raiffeisen (1818-1888). Zur Geschichte des Genossenschaftswesens vgl. die sehr ausführliche Darstellung mit Bibliographie: Faust, Geschichte und Laurinkari.

${ }^{2}$ GSTAB, Rep. 84a, Nr. 11150, Bl. 127 f. Personalbogen: Bl. 129-132. Vgl. auch DGBkA, ND-P.Z., 4. Jg., Nr. 10, Bl. 4. Das war gemäß dem oben zitierten Gründungsgesetz vom 31.7. 1895 die Hauptaufgabe der Preußenkasse. Vgl. K. Kluthe, S. 84 f.

3 Wygodzinski/Müller, S. 170. Krebs nennt die Gründung mehr „eine kulturelle und sozialpolitische Maßnahme als eine reine banktechnische Angelegenheit" (S. 563).
} 
ten vereinbaren ließ ${ }^{4}$. Die sozialpolitischen Erwägungen waren eine Folge der Industrialisierung und der damit verbundenen sozialen Mißstände, die allmählich in das Bewußtsein auch konservativerer Politiker drangen. Der Abgeordnete Hans v. Kleist-Retzow hatte im Jahre $1867 \mathrm{im}$ preußischen Herrenhaus einen Antrag eingebracht, in dem er die königliche Regierung dazu aufforderte, „zur Vermittlung des Geschäftsbetriebes der Genossenschaften [...] eine Zentralkasse ein[zu]richten und diese mit einem Betriebsfonds von 2 Millionen Talern [zu] dotieren"5. Der Antrag brachte ihm zwar von Botho Graf Eulenburg den Vorwurf des Kommunismus ein und wurde auch abgelehnt, aber ein Anfang war gemacht. Wenn man auch noch eine Zeit lang versuchte, die Wähler durch staatliche Sozialpolitik von der Sozialdemokratischen Partei und in weiterem Sinne „von jeder Art von Selbstverantwortung und Selbsthilfe“ fernzuhalten ${ }^{6}$, so ließ sich das bei zunehmender sozialer Differenzierung und wachsender Zahl von Genossenschaften und anderen sozial-reformerischen Initiativen auf die Dauer nicht durchhalten. Allmählich setzte sich auch auf seiten des Staates das Motto „Selbsthilfe ergänzt Staatshilfe"7 durch. Dies führte ab 1897 zur Gründung von Handwerkskammern und eben auch zur Gründung der Preußenkasse ${ }^{8}$, obwohl damit der Widerspruch zwischen bankwirtschaftlichen und sozialpolitischen Zielsetzungen nicht aufgehoben war, im Gegenteil, er wirkte als Stachel weiter und gab später Anlaß zu Konflikten.

Sosehr die Genossenschaften die Hilfe des Staates für den stärkeren Ausbau ihrer Krediteinrichtungen benötigten, so mißtrauisch waren sie andererseits gegenüber jeglichem zu starken staatlichen Eingriff. Wenn überhaupt staatliche Hilfe geduldet werden sollte, dann, so sagten die einen, jedenfalls nicht die des Reiches, das „weder verfassungsrechtlich die Befugnis noch verwaltungsrechtlich die nötigen Organe dafür" besitze, sondern dann nur die der „Einzelstaaten, zu deren Aufgaben ja die Fragen der Volkswirtschaftspolitik und speciell die Agrarpolitik" gehörten". Die anderen argumentierten genau umgekehrt: Wenn schon staatliche Hilfe, dann lieber die des Reiches, denn das Genossenschaftswesen sei ohnehin „im wesentlichen nicht nach Ländern [...], sondern nach Wirt-

\footnotetext{
${ }^{4}$ Die Kreditzinsen mußten niedrig gehalten, die Termine entsprechend den Bedürfnissen, vor allem der Landwirte, kurzfristig und die Haftung flexibel gehandhabt werden. Die Genossenschaften versuchten, diese Bedürfnisse ihrer Anteilseigner zu befriedigen, brauchten aber ihrerseits Banken, um sich Geld zu leihen, denn die eingezahlten Anteile der Genossenschaftler reichten bei weitem nicht aus. Die von Raiffeisen veranlaßte Resolution des Aufsichtsrats der Landwirtschaftlichen Zentral-Darlehenskasse, der späteren Raiffeisenbank, vom 14. 12. 1887 warb für die Einrichtung einer landwirtschaftlichen Reichsbank mit der Begründung, daß „die bestehende Reichsbank dem Bedürfnis des landwirtschaftlichen Personalkredits nicht genügt" (Krebs, S. 562).

${ }^{5}$ Anlage zu den Verhandlungen des Herrenhauses, Berlin 1867 und Verhandlungen vom 6. 2. 1867, S. 408 ff., zitiert bei Krebs, S. 561. Vgl. auch Busche, S. 86.

${ }^{6} \mathrm{~K}$. Kluthe, S. 82.

7 Das Wort stammt von Edmund Freiherr v. Broich, der sich für die Genossenschaften einsetzte, zitiert bei: Faust, Die Zentralbank, S. 27.

${ }^{8}$ K. Kluthe, S. 74 f. Vgl. Zur Vorgeschichte der Preußischen Central-Genossenschafts-Kasse. „Protokoll einer Sachverständigen-Anhörung am 18. Mai 1895 im Preußischen Finanzministerium zu dem Vorhaben, den genossenschaftlichen Personalkredit durch die Errichtung eines Spitzeninstituts zu fördern“, hrsg. vom Institut für bankhistorische Forschung.

${ }^{9}$ Dr. v. Miaskowski (Breslau) vor der Generalversammlung des einflußreichen „Verein[s] für Socialpolitik" am 28./29. 9. 1888 in Frankfurt/M., zitiert bei Busche, S. 86.
} 
schaftsgebieten" organisiert und „reichsgesetzlich geregelt" 10 , und auch „seine großen Zentralverbände umfaßten das ganze Reich“"1.

Dieser Konflikt zwischen Anhängern der einzelstaatlichen und der Reichshilfe für die Genossenschaften brach allerdings erst in der Endphase der Weimarer Republik offen aus, weil erst dann die politischen Differenzen zwischen Reichsregierung und preußischem Staat so tiefgreifend geworden waren, daß es nunmehr nicht nur allgemein um staatliche Unterstützung von Genossenschaften ging, sondern um die politische Richtung, aus der die Hilfe kam, um die dahinterstehenden agrarpolitischen Zielsetzungen, die natürlich bei der SPD andere waren als bei der DNVP. So stellt Krebs fest, daß seit der Ernennung Kleppers „fast alle auf die Verreichlichung der Preußenkasse gerichteten Bestrebungen, namentlich aus landwirtschaftlichen Kreisen, unter dem politischen Gesichtspunkt [standen], die Preußenkasse dem Einfluß der linksorientierten preußischen Staatsregierung nach Möglichkeit zu entziehen, wenn dies auch meistens nicht offen zugegeben" wurde" ${ }^{12}$. Wenn auch die Anhänger der Reichslösung zunächst keinen Erfolg hatten, verstummten sie nie und erhielten in der Auseinandersetzung mit dem preußischen Staat und der von ihm geführten Preußenkasse so starke Schützenhilfe von den rechtsstehenden Parteien, daß ihnen die Deutsche Genossenschaftskasse schließlich als reife Frucht in den Schoß fiel, welche sich die Genossenschaften ursprünglich so sicherlich nicht gewünscht hatten ${ }^{13}$.

Das Motto, unter das Johannes Miquel die Gründung der Preußenkasse gestellt hatte, nämlich den Genossenschaften „zur vollen Selbständigkeit $[z u]$ verhelfen und ihnen eines Tages $[z u]$ ermöglichen, die Kasse selber zu übernehmen"14, wurde zwar von allen bejaht, aber über den Weg dorthin gab es erhebliche Meinungsverschiedenheiten. Die Genossenschaften vermochten nur schwer einzusehen, daß sozialpolitische Zielsetzungen zu ihrer Durchsetzung moderner banktechnischer Strukturen bedurften und daß ein Teil ihrer Selbständigkeit aufgegeben werden mußte, um die notwendige Kraft zu einer effektiven Selbsthilfe zu erlangen. Hinzu kam, daß die diesbezüglichen Auseinandersetzungen zwischen Genossenschaften und preußischem Staat sich vor dem Hintergrund einer Agrarkrise abspielten, über deren Lösung in Preußen und im Reich kontroverse Auffassungen herrschten, die jeweils von einem Teil der Genossenschaftsvertreter geteilt wurden. Damit aber wurden aus den zunächst rein wirtschaftlichen und sozialen Problemen, welche die Preußenkasse zu lösen versuchte, eminent politische Ziele und Maßnahmen, die die Preußenkasse mitten in die scharfen agrar- und finanzpolitischen Auseinandersetzungen in der Endphase der Weimarer Republik hineinrissen.

${ }^{10}$ Das 1. preußische Genossenschaftsgesetz stammte aus dem Jahre 1867 und war Grundlage für das spätere Reichsgesetz, das schließlich in revidierter Form 1889 als Reichsgenossenschafts-Gesetz Gültigkeit erlangte (K. Kluthe, S. 86).

11 Krebs, S. 562, vgl. auch die Raiffeisen-Resolution (s.o.).

12 Krebs, S. 573.

13 Die Umwandlung der Preußenkasse in "Deutsche Zentralgenossenschaftskasse“ geschah am 21. 10. 1932 durch Notverordnung des Reichspräsidenten.

${ }^{14}$ K. Kluthe, S. 85. 


\section{Preußen nach dem Ersten Weltkrieg}

Preußen war nach dem Ersten Weltkrieg sowohl politisch, wirtschaftlich und sozial wie in seinem Verhältnis zum Deutschen Reich in eine völlig neue Lage geraten ${ }^{15}$. In den sechs preußischen Ostprovinzen und in den beiden Mecklenburg lagen „über 11 Millionen Hektar, annähernd $60 \%$ des gesamten zur Vermögenssteuer veranlagten Grundvermögens“, das sind „37,6\% des Reichsgebietes. Davon befanden sich über 5,8 Millionen Hektar, mehr als die Hälfte also, in den Händen des Großgrundbesitzes und hiervon wiederum die Hälfte, 2,9 Millionen, in den Händen von 1155 Großgrundbesitzern, die jeder mehr als 1000 ha genutzten Bodens besaßen. "Daraus läßt sich, auch ohne Mecklenburg einzubeziehen, folgendes Fazit ziehen: „Berücksichtigt man gewisse homogene Züge des ostdeutschen Großgrundbesitzes sowie Struktur und Einfluß der agrarischen Organisationen und Interessengruppen vornehmlich dieser Gebiete, so erscheint es nicht unbegründet, von einer Oligarchie und einem Bodenmonopol des Großgrundbesitzes in Ostdeutschland zu sprechen. "Hinzu kam, daß die Wirtschaftsweise trotz des technischen Fortschritts im Landbau „überaus individuell, teilweise stark traditionsgebunden geblieben" war ${ }^{16}$.

Die Klasse der Großgrundbesitzer wurde aber nicht nur durch ihre gemeinsame Lebensweise und die Standesinteressen miteinander verbunden, sondern auch durch die gemeinsame Erfahrung des verlorenen Krieges und seiner Folgen, wobei „die Abtrennung weiter Gebiete und die Abschnürung Ostpreußens von der Hauptmasse Preußens und des Reiches [...] wie starke Verletzungen“" wirkten, die die Kriegsgeneration nicht bereit war, hinzunehmen, ohne gleichzeitig "entschiedene Vorsorge für die Zukunft zu treffen“, eine Vorsorge, die sich weit mehr auf „nationale“ als auf „soziale“ Ziele richtete, ja letztere „alsbald gänzlich beiseite“ drängte ${ }^{17}$. Es geht hier um die in der Politik vertretenen sozialen Ziele; daß privat, im Rahmen der Patriarchats, soziale Verantwortung übernommen wurde, steht außer Frage. Um die teilweise reaktionäre politische Einstellung der Großgrundbesitzer, im Sinne des Versuchs, alte Verhältnisse wieder herzustellen, zu verstehen, muß man sich klarmachen, was für den Großgrundbesitz auf dem Spiele stand. „,Der Gutsherr, in der Eigenschaft eines vom Landrat und Kreisausschuß bestätigten Amtsvorstehers, übte weiterhin die Polizeigewalt in seinem Bezirk aus und verkörperte die Selbstverwaltung des Bezirks in seiner eigenen Person.' Dies war ein ,Relikt feudaler Verwaltungsstruktur, das den Gutsherren weit über ihre ökonomische Bedeutung hinaus politische Macht und den 'Hintersassen dauernde politische Unselbständigkeit zuwies.' Es handelt sich hierbei nicht etwa um eine Schilderung von Verhältnissen im frühen 19. Jahrhundert, sondern aus der Zeit

15 Vgl. die Reichsverfassung von 1871 mit der Weimarer Verfassung in Bezug auf die Stellung PreuBens im Reich und die bereits erwähnte grundlegende und informative Arbeit von Hertz-Eichenrode. Vgl. auch Schumacher, Land und Politik, sowie für die Zeit vor dem Ersten Weltkrieg: Puhle, Interessenpolitik.

${ }^{16}$ Schulz, Stützungsmaßnahmen, S. $146 \mathrm{f}$. Vgl. Boyens, S. 18 ff. Boyens schildert die Verhältnisse auf den Gütern und die mangelnde Fähigkeit, die veränderte Situation nach dem Ersten Weltkrieg zu erkennen und Konsequenzen daraus zu ziehen, aus eigener Erfahrung. Vgl. auch Görlitz, der mit starker Sympathie für die führende Schicht schreibt. Schissler, in: Puhle/Winkler, Preußen S. $89 \mathrm{ff}$. und ebenda: Berdahl, S. $123 \mathrm{ff}$.

${ }_{17}$ Schulz, Stützungsmaßnahmen, S. 163. Vgl. auch Topf, S. 65 ff. und Fiederlein, S. 6. 
der Weimarer Republik."18 Sie hatten also nicht nur Besitz und politische Macht zu verlieren, mit dem Verlust des Gutes zerbrach meist auch eine Lebens- und Arbeitsgemeinschaft und das Zentrum einer oft weit verstreuten Familie, da ja nur der älteste Sohn jeweils Erbe war ${ }^{19}$. Es bedeutete auch das Ende des Bewußtseins, neben Armee und Verwaltung, die in ihren Spitzen beide häufig aus dem Landadel rekrutiert wurden, tragende Säule des Staates zu sein ${ }^{20}$. Für Menschen, die teilweise seit Jahrhunderten so gelebt hatten, war ein solcher Zusammenbruch unvorstellbar, obwohl es auch Menschen gab, welche die neuen Zeichen der Zeit erkannt hatten, doch war es gewiß noch eine Minderheit.

Bei der Durchsetzung der standespolitischen Ziele spielten die verschiedenen Interessenorganisationen der Landwirte, besonders der Reichslandbund und die „Grüne Front“, eine zentrale Rolle21. Der Wunsch der Großagrarier, ,oligarchisch“ die eigenen Interessen zu vertreten, wurde zusätzlich gespeist durch das Mißtrauen gegen Berlin, das in folgender Äußerung des ehemaligen Oberpräsidenten von Ostpreußen, Adolf v. Batocki, recht plastisch zum Ausdruck kommt: „Von Berlin den Segen zu erwarten, wäre verfehlt. Schon das wirre Durcheinander der dortigen preußischen und Reichsinstanzen erschwert bei allem guten Willen eine wirkliche Unterstützung, und das Leben im Berliner ,Milieu' macht diesen Stellen ein verständnisvolles Eingehen auf die Bedürfnisse einer entlegenen, abgeschnürten Agrarprovinz wie Ostpreußen vollends unmöglich. Nur durch eine starke, selbständig aus eigener Verantwortung handelnde, in allen wirtschaftlichen Dingen entschlossen vorgehende Selbstverwaltung können wir die Probleme unserer Zukunft lösen." ${ }^{22}$ Dahinter steckte ein massives Mißtrauen gegen „das System“ überhaupt. Auch die „neupreußische Verwaltung“, soweit sie nicht von den eigenen Standesgenossen wahrgenommen wurde, erfreute sich keiner großen Beliebtheit. Alles war ,viel schwerfälliger und bürokratischer als früher [geworden], als man dem, freien Ermessen' des Beamten einen weit größeren Spielraum ließ“, und auch lassen konnte, weil die Verwaltungsstrukturen noch überschaubarer waren $^{23}$.

Unterstützt wurde der Wunsch nach Selbstverwaltung durch den Artikel 63 der Reichsverfassung, nach dem „die Hälfte der Vertreter Preußens im Reichsrat von den Provinzialverwaltungen gewählt und entsandt werden “ mußte, womit die Provinzialvertreter bei der Stimmabgabe im Reichsrat eine gewisse Unabhängigkeit von den Intentionen des Staatsrates erlangten. Das gab den noch 1927 aus den östlichen Provinzen ent-

\footnotetext{
${ }^{18}$ Krockow, Reise, S. 191.

19 Vgl. dazu die Erinnerungen des Fürsten zu Dohna-Schlobitten, Berlin 1989.

${ }^{20} \mathrm{Vgl}$. zu dieser Einstellung den selbst in konservativen Kreisen als extrem rechts eingeschätzten Hans v. Rohr-Demmin, in: BAK, Kleine Erwerbungen, Nr. 404, S. 64 ff. und die Erinnerungen von Elard v. Oldenburg-Januschau.

${ }^{21}$ Der Reichslandbund wurde 1893 als „Bund der Landwirte“ gegründet. Seine "hemmungslose Agitation [ließ] schon etwas von den Dämonen des kommenden Jahrhunderts ahnen" (Krockow, Reise, S. 191 f.). Vgl. auch Puhle, Interessenpolitik, und die dort angeführte Literatur sowie Topf. 22 Mit vollständigem Namen: Adolf Tortiowicz v. Batocki-Friebe, zitiert bei Baier, S. 400. Zu Batocki vgl. v. d. Groeben, in: Persönlichkeiten, S. 30.

${ }^{23}$ Topf, S. $18 \mathrm{f}$.
} 
sandten "konservativen Persönlichkeiten“, die teilweise der DNVP angehörten, einen gewissen Einfluß24.

Preußen, so unbeliebt und schließlich verhaßt seine sozialdemokratisch geführte Regierung bei den Großagrariern sein mochte, war seinerseits stark an Ostpreußen und seiner Sanierung interessiert, wofür die folgenden Ausführungen des Ministerpräsidenten Otto Braun, ein Ostpreuße mit Leib und Seele ${ }^{25}$, sprechen: „Unsere Kriegsgegner haben bei Schaffung des Korridors die Absicht gehabt, Ostpreußen verkümmern zu lassen, um es dann im geeigneten Moment zur Beute der östlichen Nachbarn werden zu lassen“, und das müsse verhindert werden. Für diese Aufgabe, die Preußen als die seine ansah, verlangte es allerdings die Hilfe des Reiches, da "Preußen am meisten durch Verlust von Land und durch schlechte Grenzziehung von den Kriegsfolgen betroffen worden "sei und vom Reich keinerlei „Ersatz bekommen hätte“26. Auch der folgende Auszug aus der Denkschrift des preußischen Landwirtschaftsministers, Heinrich Steiger, zur Siedlungsfrage ${ }^{27}$ charakterisiert die Situation Preußens gegenüber dem Reich, das sich durch die Bereitstellung finanzieller Mittel einen entscheidenden Einfluß auf die preußischen Ostgebiete zu sichern versuchte ${ }^{28}$. Steiger sah es als „Pflicht des Reiches“ an, die „erforderlichen Geldbeträge“ zu gewähren, um „Preußen in den Stand zu setzen, [die Siedlungs]Aufgaben nach den vom Reich verfassungsmäßig aufgestellten Grundsätzen zu lösen“, dagegen müßte es „als verfassungswidrig [...] bezeichnet werden, wenn das Reich an die Hergabe der zur Durchführung jener wichtigen Lebensaufgaben erforderlichen Mittel die Bedingung knüpfen wollte, nunmehr selbst jene Arbeiten in die Hand zu nehmen. Ein solches Vorgehen würde auch vom praktischen Standpunkte zu großen Unzuträglichkeiten führen, ja wahrscheinlich überhaupt unmöglich sein. " 29

Wie aus den Aufzeichnungen Hermann Pünders, Staatssekretär in der Reichskanzlei, hervorgeht, herrschte schließlich „Einvernehmen darüber, daß die gegenseitige Bekämpfung von Ressort zu Ressort durch Zeitungsartikel, gelegentliche amtliche und halbamtliche Äußerungen nunmehr ein Ende haben müsse, und daß sich die Reichs- und preußischen Ressorts wieder auf die in der gemeinsamen Kabinettssitzung vom 6. August 1926 vereinbarten Grundsätze über die Zusammenarbeit des Reichs- und Preußens in Siedlungsangelegenheiten und die darauf aufgebauten Richtlinien zurückfinden müßten “ 30 .

${ }^{24}$ Ehni, S. 15 f. Lutz Graf Schwerin v. Krosigk, der vom 2. 6. 1932 bis Kriegsende Reichsfinanzminister war, schreibt in seiner Geschichte der Finanzpolitik: Der Einfluß Preußens im Reichsrat sei geschmälert gewesen, „denn von seinen 27 Stimmen wurden nur 14 Stimmen der Staatsregierung einheitlich abgegeben. Die 13 Provinzstimmen dagegen zersplitterten sich, da die Provinzen, in Sonderheit die östlichen, politisch anders standen als die Staatsregierung und Männer wie v. Gayl-Ostpreußen, Graf Behr-Pommern, Arnim-Brandenburg sich auch von dem konzilianten Staatssekretär Weismann (SPD, PrStM), der vor jeder Abstimmung versuchte, die Provinzvertreter zu einer einheitlichen Stimmabgabe zu bewegen, nicht umstimmen ließen“ (S. 57 f.).

${ }^{25} \mathrm{Zu}$ Brauns „tiefsitzenden und urwüchsigen Patriotismus[, der] sich hauptsächlich gegen Osten hin orientierte“, vgl. Schulze, Braun, S. 675 f.

${ }^{26}$ Ministerbesprechung beim Reichspräsidenten, 21. 12. 1927, in: AdR-Marx IV, Nr. 383, S. 1187 f.

${ }^{27}$ Denkschrift des PrMfLuF. vom 3. 1. 1927: Tatsachen zur Siedlung in Preußen, zitiert bei Baier, S. 406.

${ }^{28}$ Der Reichstag hatte am 1.7. 1926 für die nächsten 5 Jahre je 50 Millionen RM für Siedlungszwecke zur Verfügung gestellt.

${ }^{29}$ So die o. g. Denkschrift, zitiert bei Baier, S. 437.

${ }^{30}$ Pünder, 2. 3. 1927, zitiert ebenda. 
Welche Möglichkeiten hatte Preußen, um sich erfolgreich gegen die Eingriffe des Reiches wehren und seine eigene Politik durchsetzen zu können? Es hatte aufgrund seines großen Gebiets- und seines hohen Bevölkerungsanteils von den Ländern die stärkste Stellung im Reich, und es stellte zusammen mit diesen auch aufgrund „seines vergleichsweise vorzüglich strukturierten, zentralistisch ausgerichteten Verwaltungsapparates, seines wirtschaftlichen Potentials und seines Steueraufkommens ein solides Gegengewicht gegen das Reich dar"31. Ein wichtiges Instrument zur Durchsetzung der preußischen Interessen war die Überlegenheit der preußischen Verwaltung gegenüber der des Reiches. So beklagte zum Beispiel der Reichsfinanzminister Lutz Graf Schwerin v. Krosigk, daß sich im Gegensatz zu der starken Stellung des preußischen Finanzministers, der das Recht hatte, finanzpolitisch in die einzelnen Ressorts einzugreifen, „im Reichsfinanzministerium noch keine feste ,Etattradition' hatte bilden können "32. Außerdem hatte der preußische Finanzminister, dem ja die Preußenkasse unterstand, im Reich ohnehin eine „Sonderstellung“, weil alle vom Reich erlassenen Verordnungen, von denen finanzielle Auswirkungen auf Preußen zu erwarten waren, im Einvernehmen mit ihm geregelt werden mußten ${ }^{33}$. Trotz der wachsenden genossenschaftlichen Anteile an der Preußenkasse waren Leitung und Aufsicht bei der Preußenkasse, also weiterhin allein beim preußischen Staat, verblieben; dieser bestimmte Präsident und Direktorium, und da sich immer mehr Genossenschaften aus dem ganzen Reichsgebiet angliederten, wuchs sein Einfluß auch im Reich ${ }^{34}$.

Nicht nur ,alle Übergriffe des Reiches, die die Verwaltungszuständigkeit Preußens betrafen“, wurden energisch zurückgewiesen, sondern auch „das Vordringen der

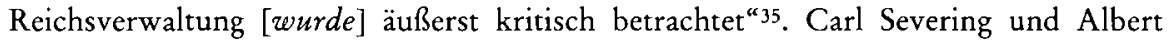
Grzesinski, die beiden wichtigsten preußischen Innenminister, sahen den besten Schutz der Republik im "Ausbau eines starken preußischen Staates“. Dazu gehörte die Demokratisierung der Verwaltung, um „unter allen Umständen zu verhindern, daß die Deutschnationalen in Preußen die Beamtenschaft, also die Verwaltung und die Exekutive in die Hand“ bekamen ${ }^{36}$. Als Grzesinski im Oktober 1926 preußischer Innenminister wurde, „war etwa $27 \%$ “ des hohen Beamtentums aristokratisch ${ }^{37}$. Während sich „auf der unteren Ebene [...] nicht viel“ änderte, war die „Demokratisierung der Verwaltung auf den höheren Ebenen erfolgreich, wurde aber von der deutschnationalen Opposition heftig kritisiert. Unter Hinweis auf das sagenhafte Idealbild vom preußischen Beamten als eines interesselosen, pflichtversessenen Staatsdieners warf sie den Regierungsparteien Pfründenwirtschaft zur Versorgung ihrer eigenen Mitglieder vor." ${ }^{\text {"38 }}$

Zum Demokratisierungsprozeß gehörte auch „der Ausbau der preußischen Polizei, um den Einfluß der Reichswehr zurückdrängen [und] für die Aufrechterhaltung von $\mathrm{Si}_{\text {- }}$

${ }^{31}$ Drei Fünftel des Reichsgebietes mit dem jeweiligen Bevölkerungerungsanteil (Ehni, S. 15 ff.).

Vgl. auch Fiederlein, S. 4 f.

32 Schwerin v. Krosigk, Staatsbankrott, S. 48.

${ }^{33}$ Ehni, S. 35.

${ }^{34}$ Faust, Zentralbank, S. 38 f. 1913 arbeiteten 7 nichtpreußische Zentralkassen, Ende 1925 schon 29 mit der Preußenkasse zusammen.

${ }^{35}$ Ehni, S. 18, 24, 36, dort wird auch die besondere Rolle des preußischen Innenministers betont.

${ }^{36}$ Grzesinski an Fritz Tejessy, 12. 2. 1925, zitiert bei Ehni, S. 39.

${ }^{37}$ Ehni, S. 47 ff. Vgl. Grzesinskis eigene Ausführungen in: Grzesinski, Lebensweg, in: BAK, Kleine Erwerbungen, Nr. 144, B1. 205 ff.

${ }^{38}$ Korff/Ranke, S. 575. 
cherheit und Ordnung [...] im Kampf gegen ,Links- und Rechtsradikalismus" sorgen zu können ${ }^{39}$. Doch auch hier lag der Erfolg vor allem auf den oberen Ebenen, auf der mittleren entstammten „1928 noch $45 \%$ der aktiven Polizeioffiziere aus dem Offizierskorps der kaiserlichen Armee" 40 . Den stärksten Widerstand der Deutschnationalen und vor allem der Großagrarier löste allerdings die von Grzesinski veranlaßte Auflösung der Gutsbezirke, jener „kommunal unabhängigen Herrschaftsbereiche“, aus, denn damit wollte die preußische Regierung mit den Worten Grzesinskis bewußt „der junkerpreußischen Reaktion, die vor allem im Osten ihre Macht in den Gutsbezirken rücksichtslos politisch auswertete, eine wichtige Waffe aus der Hand" schlagen ${ }^{41}$.

In dem Maße, in dem die Reichsregierung konservativer wurde ${ }^{42}$, das heißt unter den Einfluß der Kreise geriet, die auch den Ton im agrarischen Osten angaben, in dem Maße wuchs auch der Gegensatz zwischen Preußen und Reich. Das begann sich mit der Präsidentschaft Hindenburgs im Jahre 1925 zu verstärken ${ }^{43}$. Topf beschreibt als Zeitzeuge unter der launigen Kapitelüberschrift: „Der König ging - die Junker blieben“, wie Hindenburg „die Stellung seiner Standes- und Berufsgenossen außerordentlich gefestigt und gestärkt“ habe und daß dies "vielfach ganz unbewußt und ungewollt geschehen" sei. „Nicht in feudalen Zirkeln und nicht in irgendwelchen Parteien also ist die Macht des Junkertums konzentriert - es ist nicht einheitlich, es ist überhaupt nicht organisiert. Aber diese scheinbare Schwäche ist eben doch seine wahre Stärke. "44 Folgerichtig wurde alles, was den Einfluß Preußens und damit stabilere demokratische Strukturen förderte, bekämpft ${ }^{45}$. Das führte insofern zu einer schizophrenen Situation, als die eigentlichen ,Urpreußen' das zerstörten, was sie erhalten zu wollen vorgaben, nämlich Preußen und die durch Preußen repräsentierten Werte. Umgekehrt kämpften die Demokraten für die Erhaltung Preußens als Bastion der Demokratie, obwohl sie teilweise durchaus Anhänger einer Reichsreform waren.

\section{Die finanzielle Situation der Preußenkasse und die Verschuldungskrise in der Landwirtschaft um 1928}

Der Haupthebel zur Bezwingung Preußens war der finanzielle Druck, den das Reich zunehmend auf Preußen auszuüben versuchte, und das erste Opfer glaubte man in der Preußenkasse gefunden zu haben, da sie am stärksten von der Verschuldungskrise der ostdeutschen Landwirtschaft betroffen war. Angefangen hatte die Preußenkasse mit einem Grundkapital von 5 Millionen Mark, das aber ständig durch Ergänzungsgesetze erhöht werden mußte, zuletzt 1918 auf 125 Millionen Mark. Die Hauptaufgabe der Preu-

${ }^{39}$ Ehni, S. 36 und 39.

${ }^{40}$ Korff/Ranke, S. 575.

41 Ehni, S. 39 und 375. Es gab „nach einer Volkszählung von 1905 in den sieben östlichen Provinzen 14687 Gutsbezirke mit 1935000 Einwohnern“.

42 Die DNVP hatte bei den Dezember-Wahlen 1924 immerhin 103 Sitze im Reichstag erlangt, 1927 stellte sie gar vier Minister, nachdem die sogenannte „stille Koalition“ mit der SPD durch einen mit der Aufdeckung der Zusammenarbeit von Reichswehr und Roter Armee verbundenen Mißtrauensantrag gegen die Regierung Wilhelm Marx beendet worden war.

${ }_{43} \mathrm{Vgl}$. Jatzlauk, S. 59-68.

${ }^{44}$ Topf, S. 65 ff. Vgl. Fiederlein, S. $192 \mathrm{f}$.

${ }^{45} \mathrm{Vgl}$. als typisches Beispiel einer solchen Polemik die Rede Stapels von 1932. 
ßenkasse war, den Genossenschaftskassen „möglichst billige Kredite zu gewähren“ und „die überschüssigen Gelder verzinslich anzunehmen“46.

Bis 1918 war das Verhältnis der deutschen Genossenschaften zur Preußenkasse „zwiespältig“, weil die Schulze-Delitzschen Genossenschaften nicht mit ihr zusammenarbeiten wollten und die Preußenkasse ihrerseits nichts mit den Konsumvereinen zu tun haben mochte ${ }^{47}$. Das änderte sich mit dem Ergänzungsgesetz vom 5. September 1918, das der Preußenkasse gestattete, auch direkt an Einzelgenossenschaften verzinsliche Darlehen zu gewähren, wenn die Zentralkassen deren Kreditbedarf nicht befriedigen konnten oder „wenn von der Eingliederung der Einzelgenossenschaften in die Zentralkassen aus wirtschaftlich berechtigten Gründen abgesehen worden war “48. Das gleiche Gesetz erlaubte auch, Kredite an Unternehmen zu geben, an denen der Staat beteiligt war ${ }^{49}$, beziehungsweise Darlehens-, Landes- und Sparkassen mit Krediten zu unterstützen, bis diese dann ihre eigenen Zentralbanken schufen. Zweigstellen richtete die PreuBenkasse nicht ein, um den Grundsatz der genossenschaftlichen Selbstverwaltung nicht zu verletzen. Kein Wunder, daß sich das Verhältnis der Genossenschaften zur Preußenkasse vollständig wandelte und ihr nun Vertrauen entgegengebracht wurde ${ }^{50}$.

Das Vertrauensverhältnis verbesserte sich noch einmal, als das Gesetz vom 23. Juli 1923 den direkten Einfluß von Genossenschaftsvertretern auf die Preußenkasse möglich machte, indem jedem Inhaber einer Stammeinlage von 50000 RM eine Stimme bei der Zusammensetzung des Gesamtausschusses, den bisher nur der preußische Staat bestimmt hatte, zugestanden wurde. Der Gesamtausschuß wurde zur Unterstützung des Direktoriums eingesetzt, den Vorsitz hatte der Präsident der Preußenkasse, außerdem gehörten ihm Beauftragte verschiedener betroffener Ministerien, wie das der Finanzen und der Landwirtschaft, sowie Vertreter der Genossenschaften an. Zunächst waren es höchstens 30, ab 192050 Mitglieder, wobei ein engerer Ausschuß von 17 Mitgliedern „dem Direktorium als Beirat zur Seite stand“. Nach dem Abänderungsgesetz vom 25. Juli 1923 und der Abänderungsverordnung vom 18. Januar 1924 wurden die Ausschußmitglieder nicht mehr durch die Staatsregierung, sondern von den Anteilseignern bestellt. Seit 1924 hatte sich auch das Stimmrecht verändert: pro 25000 RM eine Stimme. Kam es bei Abstimmungen zu Stimmgleichheit, gab der Präsident den Ausschlag. „Der Ausschuß war bei der Aufstellung der Grundsätze für die Kreditgewährung und für die Annahme von Spareinlagen sowie bei der Feststellung des Jahresabschlusses gutachtlich anzuhören. Ein Mitbestimmungsrecht hatte er nicht", aber das Direktorium mußte ihn „über die Geschäftsentwicklung der Bank informieren“; diese verwaltungsmäßige Unabhängigkeit blieb auch nach 1924 erhalten $^{51}$.

Damit wurde Miquels Absicht im Ansatz verwirklicht, was auch in der Begründung des Gesetzes zum Ausdruck kam. Die praktischen Folgen des Gesetzes zeigten sich schon Ende 1924, als sich „insgesamt 53 Anteilseigner mit Stammeinlagen von zusammen 29835000 RM“ beteiligten. Ende 1925 war diese Summe bereits auf 33 Millionen

\footnotetext{
46 Faust, Zentralbank, S. $29 \mathrm{ff}$.

${ }^{47}$ K. Kluthe, S. 109. Vgl. Grünfeld, Ernst: Das Genossenschaftswesen, volkswirtschaftlich und soziologisch betrachtet, Halberstadt 1928.

48 Faust, Zentralbank, S. 31.

${ }^{49}$ Das Gesetz erhielt für Klepper große Bedeutung, als er den Scheuerkonzern kaufte.

${ }^{50} \mathrm{~K}$. Kluthe, S. $110 \mathrm{f}$.

51 Faust, Geschichte, S. 547 f. und 558.
} 
angestiegen, was nominell fast dem Anteil Preußens entsprach, aber da tatsächlich nur 13,5 Millionen eingezahlt worden waren und der Rest als Kredit ausstand, geriet die Preußenkasse in finanzielle Schwierigkeiten ${ }^{52}$.

Abgesehen davon war die finanzielle Lage der Preußenkasse auch sonst Mitte der 20er Jahre nicht rosig, denn die Nachwirkungen der Inflation hatten die Kreditgenossenschaften „entlaubt wie ein[en] von Raupen kahl gefressene[n] Baum“53 zurückgelassen. Zwar waren die Landwirte ihre Schulden in der Inflation schlagartig losgeworden, doch hatten sie auch ihre Ernten verkauft. Als dann die Reichsmark geschaffen wurde, war kein Geld in der Kasse, um die laufenden Kosten, Saatgut und Neuinvestitionen zu bezahlen. So nahm die Landwirtschaft, wie Klepper 1927/28 in einer Artikelserie zur Agrarkreditfrage in Der Volkswirt ausführte ${ }^{54}$, „Kredite um jeden Preis auf“, die ihr „gemäß der damaligen Fehlbeurteilung der Rentabilitätsmöglichkeiten unsystematisch gegeben“ wurden. Dies wirkte sich bereits bei „der Ernteverwertung" ein Jahr später insofern negativ aus, als „man glaubte, [...] eine allgemeine Produktionssteigerung könne die Entspannung bringen“, ein Glaube, der, „mit Autarkiegedanken verbunden“, zu einer „weiteren Verschuldung“ führte ${ }^{55}$. Hinzu kam, daß durch „scharfe Preisbeschneidungen bei den Erzeugnissen, enorm steigende Kosten, namentlich der Zinsen und Steuern“, und „aufeinanderfolgende Fehlernten“ die Verschuldung auf dem Lande so stark gestiegen war, daß eine Enquête des Reichsernährungsministeriums für die Wirtschaftsjahre 1924-1926 feststellen mußte, daß „über die Hälfte aller größeren und weit mehr als ein Drittel aller kleineren Betriebe mit Verlust gearbeitet haben“56. Auch „der Versuch einer Sanierung durch Steigerung des Rohertrages unter dem Zeichen der Preisschere stellte sich“, wie Klepper kritisierte, „als die zweite große Fehldisposition heraus“, denn nun wurde aus dem bei der ersten Kreditaufnahme entstandenen Substanzverlust ein „Kapitalschwund“. „Die Zusammenbrüche landwirtschaftlicher Betriebe“ häuften sich Ende 1925 „epidemisch“, und „der Güter- und Pachtmarkt“ stagnierte Anfang 1926 ${ }^{57}$.

Obwohl die Krise im Laufe des Jahres infolge einer gewissen Entspannung auf dem Geldmarkt abzuflauen schien und man die Zeit zu einer gewissen Konsolidierung der Kreditverhältnisse zu nutzen versucht hatte, würde, so prophezeite Klepper, der gleiche oben von ihm skizzierte Kreislauf erneut einsetzen, wenn das Geld knapp werde. Denn die Finanzkrise sei nicht, wie vor allem Großlandwirte gern behaupteten, die Folge der mangelnden Rentabilitätsmöglichkeiten der Landwirtschaft, sondern es sei genau umgekehrt. Insofern würde also „die aus der Landwirtschaft erhobene Forderung einer Stützungsaktion“, die Klepper angesichts der Lage für verständlich hielt, gesamtwirtschaftlich nur dann vertretbar sein, wenn das dafür investierte Kapital rentabel angelegt wür-

52 Faust, Geschichte, S. 559 f. 3 Millionen RM kamen von der „Deutschen Raiffeisenbank“, die vor 1923 "Landwirtschaftliche Zentraldarlehenskasse" hieß und erst seit 1922 mit der Preußenkasse in Geschäftsverkehr getreten war. Klepper mußte sie liquidieren (s.u.).

${ }^{53}$ Zitat aus dem Bericht der Preußenkasse (DGBkA), vgl. Faust, Zentralbank, S. 39.

${ }^{54} \mathrm{Vgl}$. Klepper, Agrarkredit I und II, Grundsätzliches zur Agrarfrage, Zum Ostprogramm, in: Der Volkswirt, und Rationelle Agrarpolitik, in: Magazin der Wirtschaft.

${ }^{55}$ Klepper, Agrarkredit I, S. 375.

${ }^{56}$ Faust, Geschichte, S. 560. Vgl. auch Sering, S. 39 ff. und 661, Fiederlein, S. 6 ff. und Topf, S. 32 ff.

${ }^{57}$ Klepper, Agrarkredit I, S. 375. 
$\mathrm{de}^{58}$. Mitte Oktober 1925 hatten die über die Preußenkasse, die Verbandskassen und die Genossenschaften laufenden, „der Landwirtschaft bereitgestellten Kredite mit 1,2 Milliarden Mark ihren Höchststand erreicht“, und das bei einem Stammkapital von rund 60 Millionen RM!59

Das zusätzliche Problem dieser Verschuldung war, daß zur Zeit der Präsidentschaft von Geheimrat Semper ca. 35000 Genossenschaften mit der Preußenkasse zusammenarbeiteten. Die Zentralkassen der landwirtschaftlichen Genossenschaften waren die Hauptkreditnehmer der Preußenkasse ${ }^{60}$. An wen diese wiederum die Kredite weitergaben, unterlag nicht der Kontrolle der Preußenkasse, was dazu führte, daß die Endkreditnehmer oft gar nicht bekannt waren. Da ja das ursprüngliche Ziel der Preußenkasse die Förderung des Genossenschaftswesens durch eine Kreditpolitik mit möglichst gleichbleibendem und niedrigem Zinssatz gewesen war, hatte der preußische Staat sich lange Jahre mit einer niedrigen Verzinsung seiner Einlagen zugunsten der Genossenschaften begnügen müssen. Gleichzeitig aber hatte sich die Zahl der Genossenschaften seit Gründung der Preußenkasse ungefähr verfünffacht. Sowohl die Reichs- wie die preußische Regierung hatten diese Kreditpolitik gefördert, weil sie sich davon eine Wiederbelebung der Landwirtschaft und auch eine Erhaltung des Genossenschaftswesens versprachen. Doch entsprechend der obigen Analyse Kleppers trat das Erhoffte nicht ein, und die Preußenkasse geriet an den Rand der Illiquidität. Kreditrückzahlungen blieben aus, die Deutsche Raiffeisenbank machte Verluste und wurde „zu einem Rückgriff auf den Rediskont bei der Reichsbank in einem bisher nicht gewohnten Ausmaß gezwungen "61. Hatten die Rediskontierungen Ende 1926 noch 97,3 Millionen RM betragen, so waren sie im November 1927 bereits bei 391,5 Millionen RM angelangt!62

Die Auswirkungen der Verschuldungskrise betrafen die Landwirtschaft allerdings in unterschiedlichem Maße. Am stärksten betroffen waren die vorwiegend auf den Verkauf von Ackerprodukten angewiesenen Großbetriebe, und zwar besonders „in Pommern, Mecklenburg und Teilen von Brandenburg“, da dort die stärksten Ernteschäden vorgekommen waren. Doch in Ostpreußen, so sah Klepper voraus, stehe ebenfalls, wenn auch

${ }^{58}$ Ebenda, S. 376. Kleppers Analyse wird bestätigt von Sering, S. 39 ff.; Topf, S. 32 ff.; v. Dietze, S. $18 \mathrm{ff}$. Fiederlein, der die komplexen Ursachen für die Verschuldungskrise der Landwirtschaft sorgfältig untersucht, beschreibt ihr Erscheinungsbild im Jahre 1927 und gibt folgende Zahlen für die durchschnittliche Verschuldung am 1.1.1928 an: , ,... in Pommern 52,5\%, in OstpreuBen 57,1\% des Einheitswertes. [...] Nach Ermittlung der Landwirtschaftskammer Stettin haben 421 Betriebe im Wirtschaftsjahr 1927/28 die Zinsen nicht mehr aus dem Reinertrag decken können." Vgl. auch Boyens, Bd. II, S. $18 \mathrm{ff}$.

${ }^{59}$ Faust, Geschichte, S. 561. Fiederlein, S. 6 ff.

${ }^{60}$ Faust, Zentralbank, S. 38: Ende 1927 entfielen „rund 80\% auf landwirtschaftliche Kreditnehmer; allein $42 \%$ der Kredite waren an Genossenschaften gegeben worden, die ihren Sitz östlich der Elbe hatten“. Nach Engelmann, S. 9, waren die landwirtschaftlichen Genossenschaften „auch numerisch die größte Gruppe. Die zweitgrößte bildeten die sogenannten gewerblichen oder städtischen (Schultze-Delitzsch-) Genossenschaften, die gleichfalls in beträchtlichem Umfang Kredithilfe in Anspruch nahmen. Weniger ins Gewicht fiel die vergleichsweise kleine Gruppe der Bau- und Siedlungsgenossenschaften. Die große vierte Gruppe, die Konsumvereine, beanspruchte in den zwanziger Jahren nicht nur keinen Kredit von der Preußenkasse, sondern unterhielt bei ihr laufend bedeutende Guthaben und trug somit zur Finanzierung des Kreditbedarfes der übrigen Genossenschaften nicht unerheblich bei."

${ }^{61}$ Faust, Zentralbank, S. 41. Sering, S. $111 \mathrm{ff}$.

${ }^{62}$ Faust, Geschichte, S. 562. Vgl. Wygodzinski/Müller, S. 178. 
„in etwas langsamerem Tempo [...] der Zusammenbruch einer weit über der normalen Ausscheidungsquote liegenden Zahl von Betrieben bevor"63. Diese wirtschaftlich besonderen Verhältnisse im Osten waren durch den verlorenen Ersten Weltkrieg extrem verschärft worden und hatten damit eine starke politische Dimension gewonnen, die um so folgenreicher wurde, als sich vornehmlich die Klasse der Großgrundbesitzer immer weniger angemessen durch die neue sozialdemokratisch geführte preußische Regierung vertreten fühlte und folglich nach Anlehnung beim Reich suchte. Somit trug auch die Verschuldungskrise im Osten, durch die die Preußenkasse immer stärker unter Druck und damit in immer größere Abhängigkeit von der finanziellen Unterstützung durch das Reich geriet, dazu bei, das Verhältnis zwischen Preußen und dem Reich zu verschlechtern.

Infolge der skizzierten Umstände war die Preußenkasse im Laufe des Jahres 1927 in erhebliche finanzielle Schwierigkeiten geraten, so daß es im Reichstag bereits im März 1927 zu einer ersten Anfrage nach einer eventuellen „Verreichlichung“ der Preußenkasse gekommen war. Dies hatte der damalige Reichswirtschaftsminister Julius Curtius zwar abgelehnt, allerdings mit dem Hinweis verknüpft, daß man mit Preußen verhandle, um „dem Reich eine Vertretung im großen Ausschuß der Preußenkasse zu verschaffen"64. Auch im preußischen Landtag gab es Mitte Dezember 1927 Kritik an der Kreditpolitik der Preußenkasse. Sie gipfelte in dem Vorwurf des Sozialdemokraten Ernst Heilmann, „bei der Kreditgewährung sei der Großgrundbesitz bevorzugt worden"65. Auch die Presse nahm sich des Themas an, was schließlich das preußische Finanzministerium veranlaßte, eine Treuhandgesellschaft mit der Prüfung der Geschäftslage der Preußenkasse zu beauftragen ${ }^{66}$. Aus dieser Untersuchung ergab sich unter anderem, daß die Preußenkasse in erhebliche Liquiditätsschwierigkeiten geraten war. Der Präsident der Preußenkasse, Geheimrat Semper, wurde beschuldigt, „er habe die Situation leichtfertig herbeigeführt“, und der preußische Finanzminister Hermann HöpkerAschoff nahm im preußischen Landtag kein Blatt vor den Mund, als er über die "verschlossene Natur" Sempers und die außerordentlich schwierige Zusammenarbeit mit ihm sprach und schließlich das Fazit zog, er zeige sich „seinen Aufgaben nicht gewach$\operatorname{sen}^{\text {“ } 67}$.

Die preußische Staatsregierung handelte und entzog die Preußenkasse fürs erste dem Zugriff des Reiches, womit „das politische Preußen zum ersten und einzigen Male seine Kraft ausgenutzt und seine Initiative angespannt [batte], um auf politischem und agrarpolitischem Gebiete dem ostelbischen Großgrundbesitz eine entscheidende Position zu entreißen: es hatte die Gutsbezirke aufgehoben, und es hatte die Preußenkasse, sein Agrarinstitut, reorganisiert und als agrarpolitisches Instrument aktiviert" ${ }^{\text {"68. }}$.

\footnotetext{
${ }^{63}$ Klepper, Agrarkredit I, S. 376.

${ }^{64}$ Krebs, S. 572, zitiert: Verhandlungen des Reichstags vom 14. 3. 1927, 3. Wahlperiode, Bd. 392, Sp. 9447, 9465 und 9492.

${ }^{65}$ Krebs, S. 572, zitiert: Sitzungsbericht des PrLT, 2. Wahlperiode, Bd. 15, Sp. 22714.

${ }^{66}$ Der von Faust als nicht mehr vorhanden bezeichnete Bericht der Treuhandgesellschaft liegt in den noch weitgehend unerschlossenen Akten der Preußenkasse (Faust, Geschichte, Anm. 23, S. 721). Die Treuhandberichte für die Jahre 1928-30 (3 Berichte: März und Dezember 1929 und Dez. 1930) sind zu finden unter GSTAM, Rep. 151 IC, Nr. 10366/67.

67 PrLT, 366. Sitzung, 26. 3. 1928, Sp. 26094.

68 Topf, S. 49.
} 
Vor diesem Hintergrund fällt auf, wie unterschiedlich der notwendig gewordene Wechsel in der Führung der Preußenkasse in der wissenschaftlichen Literatur beurteilt wird. Faust schreibt: „Die preußische Staatsregierung nahm diese von politischen Gruppen geschürten, nicht aus der genossenschaftlichen Wirtschaft kommenden Vorhaltungen zum Anlaß, in der Führung der Anstalt einen Wechsel eintreten zu lassen. [...] Inwieweit für diesen Schritt der damals links gerichteten preußischen Regierung in der Tat letztlich politische Gründe eine Rolle gespielt haben, läßt sich heute schwer nachweisen. Semper selbst, der konservativ eingestellt war, hat dies jedenfalls glaubhaft zu machen versucht. “69 Dagegen bestreitet Fiederlein, daß Semper die Vorwürfe „zu entkräften vermochte, [...] denn die Leitung der Preußenkasse hatte den veränderten Kreditverhältnissen der Nachkriegszeit zu wenig Beachtung geschenkt und deshalb zu lange einen falschen Kurs in der Kreditgewährung gesteuert "70. Doch ging es ja nicht nur um Kreditgewährung; Engelmann bringt das Problem auf den Punkt: „Ein staatliches Institut, das gesetzlich wie wirtschaftlich über die erforderlichen Machtvollkommenheiten verfügte, um im Sinne der Regierungspolitik eingreifen zu können, hatte sich gerade durch diejenigen Kräfte immobilisieren lassen, die wirtschaftlich von ihm abhängig waren, es aber verstanden hatten, die Kräfte der Opposition für sich zu nutzen. Die relative Stärke der Schuldner, die mit dem drohenden Ausbruch politisch untragbarer Katastrophen in ihren Reihen gegen mögliche Kreditrestriktionen oder organisatorische Eingriffe in ihre genossenschaftlichen Selbstverwaltungsrechte zu protestieren pflegten, hatte dazu geführt, daß der Gläubiger - praktisch ein Organ der Regierung es nicht gewagt hatte, selbst gegen offensichtliche Mißstände in den Reihen der Schuldner, Desorganisation, Überschuldung, Unwirtschaftlichkeit, einzuschreiten. Er hatte so ein agrarpolitisches System unterstützt, das die Autorität des Staates untergrub. "71

Erwin Topf legte mit seiner sarkastischen Skizze über die Zustände in der Preußenkasse vor Kleppers Ernennung wieder den Finger auf die Hauptwunden: „Die Preußenkasse [...] war bis zur Jahreswende 1927/28 ein wahres Idyll altpreußischer Beamtenherrlichkeit, unberührt von den Stürmen der, großen' Agrarpolitik. Der Präsident Semper (auf Lebenszeit angestellt, unkündbarer Vertrag) war der Meinung, daß die Landwirtschaft in der Nachinflationszeit vor allem Kredite, große Kredite und nochmals Kredite haben müßte, und sei es selbst auf Kosten der eigenen Liquidität. Und er handelte dementsprechend. Sein Direktorium, in dem die Freunde des Altreichskanzlers Michaelis vom Christlichen Verein Junger Männer das Übergewicht hatten, schloß sich in allen Dingen der Meinung seines Herrn Präsidenten an. Die Aufsichtsbehörde, in Gestalt des preußischen Finanzministers, saß nebenan im Kastanienwäldchen, nur durch eine Straßenbreite von der Preußenkasse getrennt ${ }^{22}$. Und ringsumher tiefer, von keinerlei schweren Träumen beschatteter Friede"73, was selbst Faust, der sich stark um eine Verteidigung Sempers bemüht, bestätigt: „Nach einer Entwicklung von über dreißig Jahren schien die Preußische Zentralgenossenschaftskasse schon ein Wirtschaftsorganis-

\footnotetext{
${ }^{69}$ Faust, Zentralbank, S. 41, Carl Semper, Entgegnung auf den Bericht der Treuhandgesellschaft, Berlin, vom 3.2. 1928 (DGBkA).

70 Fiederlein, S. 32. Zu ähnlichem Schluß kommt Boyens, Bd. 1, S. 225 und Bd. 2, S. 173.

${ }^{71}$ Engelmann, S. $13 \mathrm{f}$.

72 Die Preußenkasse lag "hinter dem Zeughaus, unmittelbar an der Spree, aber noch südlich der Spreeinsel“" (Achterberg, S. 79).

${ }^{73}$ Topf, Napoleon, S. $466 \mathrm{ff}$.
} 
mus geworden zu sein, der ein eigenes Leben führte, dessen Geschäfte sich vielfach auf eingelaufenen Geleisen bewegten, so daß die Führung durch den Präsidenten nicht mehr so ausdrucksvoll in Erscheinung trat. "74

Es bestand kein Zweifel, mit der Ernennung Kleppers zum Präsidenten endete für die Preußenkasse das Schattendasein, es begann eine politisch unruhige Zeit. Klepper selbst hat diesen Umbruch erläutert: „Über die Behandlung der Krise des Großgrundbesitzes bestanden von vornherein lebhafte Meinungsverschiedenheiten zwischen der Reichsregierung und der preußischen Staatsregierung. Die Reichsregierung zielte darauf ab, durch generell ausgeworfene Subventionen eine allgemeine Entschuldungsaktion durchzuführen. Die preußische Staatsregierung zog den Gedanken einer bäuerlichen Agrarreform im Osten vor. Wesentlicher Exponent der preußischen Konzeption war die Preußische Zentralgenossenschaftskasse. Die Angriffe auf sie setzten im Jahre 1928, unmittelbar nach meiner Ernennung zum Präsidenten der Preußenkasse, ein. “75 Faust kommentiert: „Klepper ist nicht ganz vier Jahre Präsident der Preußenkasse gewesen. [...] Aber diese kurze Epoche hat genügt, die Preußenkasse in Kleppers Geist umzuprägen. "76

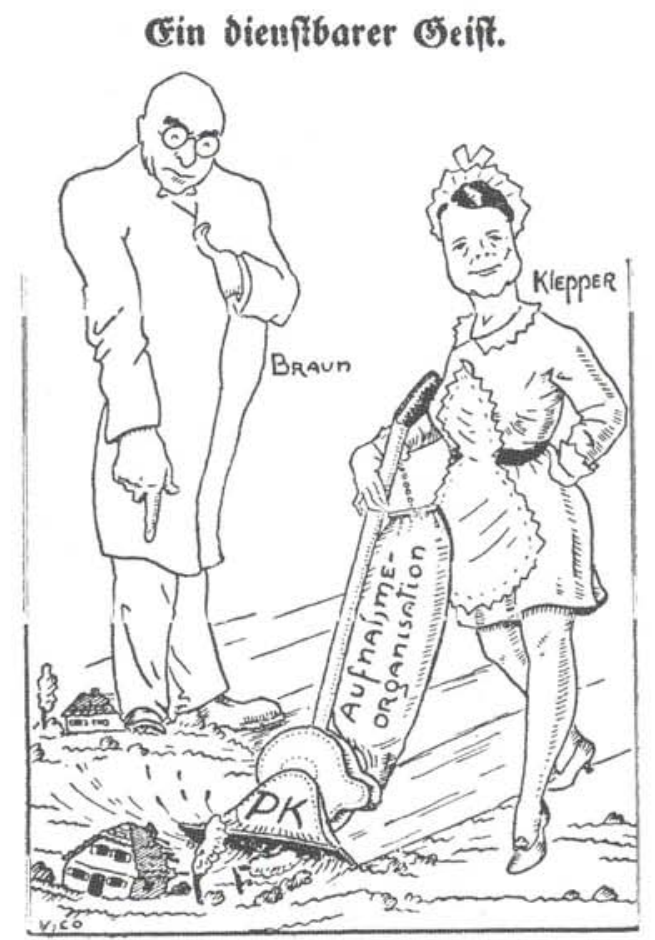

Quelle: Privatarchiv d. Verf.

\footnotetext{
${ }^{74}$ Faust, Geschichte, S. 565.

${ }^{75}$ Klepper beantwortete Fragen des Entschädigungsamtes Berlin in der Wiedergutmachungssache Emil Pahlke (Verwaltungsrechtsrat in der P.Z.) am 4. 4. 1955 (K/Kor.).

${ }^{76}$ Faust, Zentralbank, S. 41.
} 